\title{
Warum die Deutschen ihrem Abgeordneten nicht mehr vertrauen als dem Bundestag
}

\author{
Oscar W. Gabriel unter Mitarbeit von Lisa Schöllhammer
}

\section{Das Untersuchungsproblem: Vertrauen zum Parlament und zu den Abgeordneten in der parteienstaatlichen Demokratie}

Auf dem Höhepunkt der tiefen Vertrauenskrise der politischen Institutionen der USA in der zweiten Hälfte der 1970er Jahre stellte Richard Fenno die These auf, die amerikanischen Bürger liebten ihre Abgeordneten sehr viel mehr als den Kongress. ${ }^{1}$ Mit repräsentativen Umfragedaten aus den Jahren 1968 und 1977 bestätigten Glenn Parker und Roger Davidson diese These ${ }^{2}$, und knapp zwanzig Jahre später lieferten John Hibbing und Elisabeth TheissMorse $^{3}$ zusätzliche Belege für das geringe Vertrauen der US-Bürger zum Kongress und die dafür maßgeblichen Gründe: „Congress embodies practically everything Americans dislike about politics. It is large and therefore ponderous; it operates in a presidential system and is therefore independent and powerful; it is open and therefore disputes are played out for all to see; it is based on compromise and therefore reminds people of the disturbing fact that most issues do not have right answers. Much of what the public dislikes about Congress is endemic to what a legislature is. Its perceived inefficiencies and inequities are there for all to see." 4

Für die vergleichende Politikwissenschaft stellt sich die Frage, ob das beschriebene Muster des Vertrauens der Bürger zu den politischen Institutionen und Akteuren zu den zahlreichen Spezifika der Politischen Kultur der Vereinigten Staaten gehört oder ob in anderen Demokratien ähnlich divergierende Vertrauensbeziehungen der Bürger zum Parlament und zu den Abgeordneten sowie für diese Divergenz maßgebliche Motive anzutreffen sind. Die Bedeutung dieser Frage für die vergleichende Einstellungsforschung ergibt sich nicht zuletzt aus einem Vergleich der Rolle des Parlaments und der Abgeordneten im präsidentiellen Regierungssystem der USA mit den Gegebenheiten in den parlamentarischen Parteiendemokratien Europas wie der Bundesrepublik Deutschland. ${ }^{5}$ Die in den Vereinigten Staaten existierenden institutionellen Arrangements schaffen Anreize für eine starke Orientierung der Abgeordneten auf ihre lokale Wählerschaft, die in den europäischen Parteiendemokratien in dieser Form nicht vorliegen. Die daraus resultierende enge Vertrauensbeziehung zwischen den Abgeordneten und ihrer lokalen Basis wird vermutlich durch die von Seymour

1 Vgl. Richard Fenno, If, as Ralph Nader Says, Congress is 'The Broken Branch', How Come we Love our Congressmen so much?, in: Norman J. Ornstein (Hrsg.), Congress in Change: Evolution and Reform, New York 1975, S. 286.

2 Vgl. Glenn R. Parker / Roger H. Davidson, Why Do Americans Love their Congressmen so much more than their Congress?, in: Legislative Studies Quarterly, 4. Jg., (1979), H. 1, S. 52 - 61.

3 John R. Hibbing / Elisabeth Theiss-Morse, Congress as Public Enemy. Public Attitudes Towards American Public Institutions, Cambridge 1995, S. 31 - 36.

4 John R. Hibbing / Elisabeth Theiss Morse, a.a.O. (Fn. 3), S. 60.

5 Vgl. Uwe Thaysen / Roger H. Davidson / Robert G. Livingston (Hrsg.), US-Kongress und Deutscher Bundestag. Bestandsaufnahmen im Vergleich, Opladen 1988. 
Martin Lipset als „American Exceptionalism“ beschriebene kulturelle Tradition verstärkt. Die für das Verhältnis der US-Bürger zum Staat typischen Merkmale des Individualismus und Anti-Institutionalismus ${ }^{6}$ finden in der europäischen Kultur keine Entsprechung oder sind in Europa zumindest weniger stark ausgeprägt. Vor diesem Hintergrund beschäftigt sich dieser Beitrag mit der Frage, ob die in den USA gewonnenen Erkenntnisse über das Vertrauen der Bürger zum Parlament als Institution und zu den Wahlkreisabgeordneten auch für andere politische Systeme gelten, in denen die Abgeordneten durch ein Mehrheitsvotum der lokalen Wählerschaft in das nationale Parlament entsandt werden.

Auf Grund einiger Merkmale seines politischen Systems eignet sich Deutschland für eine Untersuchung dieser Art. Es gehört zu denjenigen Demokratien, in denen die Hälfte der Abgeordneten ihr Mandat einem Mehrheitsvotum der lokalen Basis verdankt und in denen die lokalen Parteiorganisationen über eine relativ große Autonomie bei der Nominierung der Wahlkreiskandidaten verfügen. Selbst die Absicherung der meisten Wahlkreiskandidaten auf den Landeslisten ihrer Parteien macht eine starke Präsenz der Abgeordneten in ihren Wahlkreisen nicht überflüssig. ${ }^{7}$ In der deutschen Parteiendemokratie mögen die Abgeordneten stärker als Repräsentanten ihrer Partei wahrgenommen werden als in den USA; dennoch präsentieren sie sich ihrer lokalen Wählerschaft als eigenständige Akteure und werben um Unterstützung für ihre Person, nicht allein für ihre Partei. Zwischen Deutschland und den USA bestehen einerseits hinreichende strukturelle Übereinstimmungen, die eine Auseinandersetzung mit der Frage, ob die Bürger die Wahlkreisabgeordneten positiver bewerten als die Institution des Deutschen Bundestages, sinnvoll erscheinen lassen. Andererseits existieren institutionelle und kulturelle Differenzen zwischen den beiden Ländern, die der Annahme jeweils unterschiedlich gearteter Vertrauensbeziehungen zwischen den Bürgern, dem Parlament und den Abgeordneten eine hohe Plausibilität verleihen.

Da sich die Untersuchung auf ein Land beschränkt, liefert sie keine systematische, kausale Erklärung der Bedeutung institutioneller und kultureller Faktoren für das Vertrauen der Bürger zur Politik. Sie beschränkt sich darauf zu prüfen, ob das in den USA ermittelte Vertrauensgefälle zwischen dem Parlament und den Abgeordneten auch unter den andersartigen institutionellen und kulturellen Bedingungen Deutschlands existiert.

Als wichtigste Datengrundlage dient eine im Frühjahr 2005 bundesweit durchgeführte Repräsentativbefragung, in der detaillierte Daten über einige in diesem Kontext interessierende Aspekte des Verhältnisses der Bürger zur Politik erhoben wurden. ${ }^{8}$ Diese Daten erlauben nicht allein einen Vergleich des Vertrauens der Bundesbürger zu den Wahlkreisabgeordneten und zum Bundestag, sondern sie liefern zusätzlich Informationen über mögliche Bestimmungsfaktoren des Vertrauens.

6 Vgl. Seymour Martin Lipset, American Exceptionalism. A Double-Edged Sword. New York / London 1996.

7 Vgl. Bernhard Weßels, Abgeordnete und Bürger. Parteien und Wahlkreiskommunikation als Faktoren politischer Repräsentation, in: Hans-Dieter Klingemann / Richard Stöss / ders. (Hrsg.), Politische Klasse und politische Institutionen. Probleme und Perspektiven der Elitenforschung, Opladen 1991, S. 348 - 355.

8 Es handelt sich um die von EMNID im Auftrag der Deutschen ESS-Gruppe durchgeführte Umfrage „Bürger und Gesellschaft“ ( $\mathrm{N}=2315$, Alte Bundesländer: 1850, Neue Bundesländer: 465). Die Erhebung wurde als CAPI durchgeführt, die Stichprobenziehung erfolgte nach dem ADM Master Sample. 


\section{Warum stehen die Abgeordneten in den USA in höherem Ansehen als der Kongress, und lassen sich diese Konstellationen auf Deutschland übertragen?}

Den Beginn der in den USA geführten Debatte über die Unterschiede im Vertrauen der Bürger zum Kongress und zu den Abgeordneten markierte ein von Fenno verfasster Essay, in dem er diese divergierenden Vertrauensbeziehungen beschrieb und einige Gründe für das vermeintliche Paradox nannte, dass die amerikanische Öffentlichkeit die Mitglieder einer Institution besser bewertet als die Institution selbst. Er führte diesen Sachverhalt auf die Verwendung unterschiedlicher Kriterien bei der Beurteilung beider Objekte zurück, wobei die für die Urteilsbildung über die Abgeordneten maßgeblichen Standards weniger anspruchsvoll seien als die Maßstäbe, an denen der Kongress als Institution gemessen werde. ${ }^{9}$ Parker und Davidson nahmen diese Überlegungen auf und konnten sie in einer empirischen Analyse bestätigen. Wie sie herausfanden, lassen sich zwischen den für die Bewertung des Kongresses und der Wahlkreisabgeordneten maßgeblichen Kriterien kaum Parallelen finden. ${ }^{10}$ Das Ansehen des Kongresses in der Bevölkerung werde durch die Wahrnehmung seiner innenpolitischen Leistungen, seiner Beziehung zur Exekutive beziehungsweise zum Präsidenten sowie des Stils und der Geschwindigkeit des Gesetzgebungsprozesses geprägt. Aufgrund der überwiegend negativen Bewertung dieser Aspekte der Arbeit des Kongresses sei auch sein Ansehen in der Bevölkerung schlecht. Für die Wahlkreisabgeordneten kämen dagegen Kriterien zur Anwendung, die sie in einem guten Licht erscheinen ließen. Mehr als 70 Prozent der Befragten stützten ihr Urteil auf die von den Abgeordneten erbrachten Leistungen für die Bürger im Wahlkreis (constituency service) und auf deren Persönlichkeitsmerkmale. Die positive Einschätzung dieser Eigenschaften führe zu einem günstigen Urteil über die Abgeordneten. ${ }^{11}$ Zwei Faktoren sind nach Parker und Davidson für das im Vergleich mit dem Kongress als Institution positivere Image der Abgeordneten ausschlaggebend: erstens die Verwendung unterschiedlicher Kriterien für die Beurteilung beider Objekte und zweitens das bessere Abschneiden der Abgeordneten auf den für die Bewertung ihrer Arbeit maßgeblichen Dimensionen.

Anders als Parker und Davidson verglichen Hibbing und Theiss-Morse das Ansehen des Kongresses mit dem der Kongressabgeordneten als Gruppe und kamen dabei zu anderen Ergebnissen als die Vorläuferstudien. ${ }^{12}$ Ihren Annahmen zufolge vermögen die Bürger zwischen der Bewertung der Abgeordneten als Gruppe und dem Kongress als Institution zu unterscheiden, zudem seien für die Bewertung beider Objekte jeweils unterschiedliche Faktoren maßgeblich. ${ }^{13}$ Dieser Wechsel der Referenzobjekte hatte eine grundlegende Veränderung der Einstellungsverteilung zur Folge, denn nunmehr schnitt der Kongress im Urteil der Öffentlichkeit besser ab als die Abgeordneten. Auch die Studie von Hibbing und TheissMorse lieferte Hinweise auf die Verwendung unterschiedlicher Standards bei der Bewertung des Kongresses als Institution und seiner Mitglieder als Gruppe. ${ }^{14}$ Diese Folgerung leiteten die Autoren allerdings nicht aus einer Auswertung offener Fragen im Rahmen einer Reprä-

9 Vgl. Richard Fenno, a.a.O. (Fn. 1).

10 Vgl. Glenn R. Parker / Roger H. Davidson, a.a.O. (Fn. 2), S. 56.

11 Vgl. ebenda, S. 59.

12 Vgl. John R. Hibbing / Elisabeth Theiss Morse, a.a.O. (Fn. 3), S. 43. Ihre Analyse bestätigt, dass Bürger ihre Wahlkreisabgeordneten besser bewerten als die Kongressmitglieder als Gruppe.

13 Vgl. ebenda, S. $42-46$.

14 Vgl. ebenda, S. $90-103$. 
sentativbefragung, sondern aus den Ergebnissen von Regressionsanalysen sowie der Auswertung von Gesprächen mit Fokusgruppen ab. ${ }^{15}$

Eine Synthese der Ergebnisse der vorgestellten amerikanischen Studien macht eine Modifikation der eingangs vorgestellten Annahme über das Vertrauen der US-Bürger zum Kongress und zu den Abgeordneten notwendig. Offensichtlich unterscheiden die Amerikaner nicht nur zwischen den Abgeordneten und dem Kongress als Institution, sondern auch zwischen den Wahlkreisabgeordneten und den Abgeordneten als Gruppe. Die größte Wertschätzung empfinden sie für die Wahlkreisabgeordneten, die geringste für die Abgeordneten als Gruppe. Der Kongress als Institution rangiert dazwischen.

Auch wenn es bisher kaum gesicherte empirische Befunde über die Rolle politischer Institutionen als Bestimmungsfaktoren politischer Einstellungen gibt, ist die Annahme, dass institutionelle Arrangements das Verhältnis der Menschen zur Politik beeinflussen ${ }^{16}$, plausibel. Die unterschiedlichen institutionellen und kulturellen Ausgangsbedingungen in den USA und Deutschland legen deshalb die Frage nahe, ob in den parlamentarischen Parteiendemokratien Europas andersartige Vertrauensbeziehungen zwischen den Bürgern, den Abgeordneten und dem Parlament existieren als in den USA.

Die für das politische System der USA charakteristische Ausgestaltung der Abgeordnetenrolle bringt eine enge Bindung der Gewählten an ihre Basis hervor und schafft damit gute Voraussetzungen für das Entstehen einer Vertrauensbeziehung zwischen den Abgeordneten und ihrer lokalen Wählerschaft. Auf Grund der kurzen Wahlperiode des Repräsentantenhauses, des Verfahrens der Direktwahl, der schwachen Position der Parteien und der lokalen Ausrichtung der Medienberichterstattung hängt die politische Karriere der Abgeordneten in hohem Maße von ihrer Unterstützung durch die Bürger im Wahlkreis ab. Daher weisen die Abgeordneten der Arbeit vor Ort eine hohe Priorität zu und bemühen sich darum, durch intensives Engagement den Erwartungen ihrer lokalen Basis zu entsprechen. Folglich wird die perzipierte Wahlkreisarbeit - neben den persönlichen Eigenschaften der Abgeordneten - zur wichtigsten Grundlage ihrer Popularität, und die ideologische oder programmatische Position spielt nur ausnahmsweise eine Rolle. ${ }^{17}$

Im politischen System Deutschlands herrschen gänzlich andere Bedingungen vor. Die Wahlperiode des Bundestages ist doppelt so lang wie die des US-amerikanischen Repräsentantenhauses. Selbst für die Direktkandidaten in den Wahlkreisen induziert das Wahlverfahren eine wesentlich stärkere Abhängigkeit von den innerparteilichen Nominierungsverfahren, als es in den USA der Fall ist. Auf die Karriereperspektiven der Bundestagsabgeordneten üben die Parteiorganisationen von der Kommunal- über die Landes- bis zur Bundesebene einen wesentlich größeren Einfluss aus als die lokale Wählerschaft. In Deutschland hängt der Mandatsgewinn entweder von der Platzierung auf der Landesliste der Parteien ab oder/und von der Nominierung durch die jeweiligen Orts- und Kreisverbände der Parteien ${ }^{18}$; über die Rolle der Abgeordneten in den parlamentarischen

15 Vgl. ebenda.

16 Vgl. Robert Rohrschneider, Learning Democracy. Democratic and Economic Values in Unified Germany, Oxford 1999.

17 Vgl. Glenn R. Parker / Roger H. Davidson, a.a.O. (Fn. 2), S. 56 - 58; Gary C. Jacobson, The Politics of Congressional Elections, New York 1997, S. 112 - 116.

18 Vgl. Suzanne S. Schüttemeyer / Roland Sturm, Der Kandidat - das (fast) unbekannte Wesen: Befunde und Überlegungen zur Aufstellung der Bewerber zum Deutschen Bundestag, in: ZParl, 36. Jg. (2005), H. 3, S. $539-553$. 
Arbeitszusammenhängen und Machtstrukturen entscheiden die Fraktionen. Dadurch entsteht im Vergleich mit den USA erstens eine stärker ausgeprägte Orientierung der deutschen Abgeordneten auf ihre Parteien und zweitens auf die nationale Politik, was durch eine weitgehend national ausgerichtete Medienberichterstattung zusätzlich gefördert wird. Das Zusammenspiel dieser Faktoren dürfte es mit sich bringen, dass die Parlamentarier in Deutschland eine schwächere Bindung an ihrer lokalen Basis entwickeln als in den USA. Sie sind der Wählerschaft weniger bekannt, werden enger mit einer Partei assoziiert und können schon aus diesen Gründen schlechter als ihre amerikanischen Kollegen eine von parteipolitischen Bindungen unabhängige, persönliche Vertrauensbasis aufbauen. Selbst wenn dies von Fall zu Fall gelingt, ist es kein konstitutives Merkmal des politischen Systems der Bundesrepublik. Auf Grund der starken Position der Parteien als intermediäre Institutionen schreiben die Wähler vermutlich auch bestimmte Leistungen für den Wahlkreis weniger dem jeweiligen Abgeordneten als seiner Partei zu. Diejenigen Faktoren, die in den Vereinigten Staaten eine positivere Einstellung zu den Parlamentariern als zum Parlament als Institution begünstigen, sind in Deutschland nicht vorhanden. Insofern ist zu vermuten, dass deutsche Bürger ihren Abgeordneten weniger vertrauen als US-Bürger den ihren.

Aus dem für parlamentarische Parteiendemokratien typischen Verhältnis zwischen Regierung und Parlament ergeben sich weitere Unterschiede zwischen den USA und Deutschland, die sich mit hoher Wahrscheinlichkeit auf das Vertrauen der Bürger zu den Abgeordneten und dem Parlament als Institution auswirken. In parlamentarischen Demokratien geht die Regierung aus dem Parlament hervor und wird vom Vertrauen der Parlamentsmehrheit getragen. Diese arbeitet eng mit der Regierung zusammen und hat kein Interesse daran, die Regierungsarbeit zu erschweren und die Regierung öffentlich zu kontrollieren und zu kritisieren. Als Gegenpol zu der aus den Mehrheitsfraktionen des Parlaments und der Regierung gebildeten Handlungseinheit fungiert die parlamentarische Opposition. Die als „Neuer Dualismus“ charakterisierte Macht- und Aufgabenteilung ${ }^{19}$ kann als Fortsetzung des Parteienwettbewerbs in der parlamentarischen Arena verstanden werden, die nur unter der Bedingung einer relativ strikten Fraktionsdisziplin funktioniert. Dies schränkt den parlamentarischen Handlungsspielraum der einzelnen Abgeordneten ein und führt dazu, dass diese von den Bürgern primär als Mitglieder von Fraktionen wahrgenommen werden.

Die Sicht der Öffentlichkeit auf die Parlamentsarbeit reflektiert den Neuen Dualismus, konfligiert aber nach Werner Patzelt mit einer immer noch am traditionellen Gewaltenteilungsmodell orientierten Erwartungshaltung der Bürger gegenüber dem Parlament. In dieser Diskrepanz von unterstellter Verfassungsnorm und perzipierter Verfassungswirklichkeit, die Hibbing und Theiss-Morse auch für die USA konstatierten, sieht Patzelt die Basis für eine kritische Bewertung der Arbeit des Deutschen Bundestages. ${ }^{20}$ Es entspräche der Funktionslogik der parlamentarischen Parteiendemokratie, wenn die Deutschen eine enge Verbindung zwischen dem Parlament und den Parlamentariern herstellten und beide Objekte nicht unabhängig voneinander bewerteten.

19 Vgl. Wolfgang Rudzio, Das politische System der Bundesrepublik Deutschland, Wiesbaden 2006, S. $197-214$, S. 202.

20 Vgl. Werner J. Patzelt, Ein latenter Verfassungskonflikt? Die Deutschen und ihr parlamentarisches Regierungssystem, in: Politische Vierteljahresschrift, 39. Jg. (1998), H. 4, S. 425 - 457. Ähnlich für die USA John R. Hibbing / Elisabeth Theiss-Morse, a.a.O. (Fn. 3), S. 87 - 90. 
Während die institutionellen Regeln der parlamentarischen Parteiendemokratie eine übereinstimmende Bewertung des Parlaments und der Abgeordneten begünstigen, wirken die europäischen Politiktraditionen zu Gunsten der Institution und zu Ungunsten der Akteure. Die von Lipset dem „Amerikanischen Exzeptionalismus“ zugerechneten Merkmale des Individualismus und Anti-Etatismus sind der europäischen, insbesondere der deutschen Politiktradition fremd. Das politische Denken der Deutschen ist traditionell staatszentriert. Anders als in den USA ist es durch den Respekt vor staatlichen Institutionen und die Verachtung für (Partei-)Politiker geprägt. Der Staat und seine Institutionen gelten als Repräsentanten des Allgemeinwohls, Parteien und Politiker als Vertreter von Partikularinteressen. Auch wenn diese Vorstellungsmuster sich seit dem Ende des Zweiten Weltkrieges abgeschwächt haben, sind sie keineswegs verschwunden. ${ }^{21}$ Ihr Fortbestand begründet die Annahme, dass die Bundesbürger - im Gegensatz zu den Amerikanern - dem Parlament mehr vertrauen als den Abgeordneten.

\section{Das Vertrauen der Bundesbürger zum Bundestag und zu den Abgeordneten}

Die folgende Untersuchung kann mangels entsprechender Daten nicht als deutsch-amerikanischer Vergleich durchgeführt werden. Wie in anderen einschlägigen Studien werden die Einstellungen der Deutschen zum Bundestag und seinen Abgeordneten analysiert und die Ergebnisse mit denen der US-amerikanischen Forschung verglichen. ${ }^{22}$ Dabei wird die Analyse auf diejenige Einstellungsdimension eingegrenzt, die in der international vergleichenden Forschung über das Verhältnis der Bürger zu den politischen Institutionen und Akteuren die wichtigste Rolle spielt: das politische Vertrauen.

\subsection{Niveau und Entwicklung}

Für Deutschland liegen nur wenige empirische Untersuchungen zu den Einstellungen der Bürger zum Parlament und zu den Abgeordneten und noch weniger detaillierte Vergleiche des Vertrauens zu diesen Akteuren vor. ${ }^{23}$ Allerdings enthalten zahlreiche seit den $1970 \mathrm{er}$

21 Vgl. Martin Greiffenhagen / Sylvia Greiffenhagen, Ein schwieriges Vaterland. Zur politischen Kultur im vereinigten Deutschland, München 1993, S. 73 - 85, S. 174 - 188; Everhard Holtmann, Die Deutsche Tradition und das politische System der Gegenwart, in: Oscar W. Gabriel / Everhard Holtmann (Hrsg.), Handbuch Politisches System der Bundesrepublik Deutschland, München / Wien 2005, S. 5 - 41.

22 Vgl. Samuel C. Patterson / John Wahlke / Robert Boynton, Dimensions of Support in Legislative Systems, in: Allan Kornberg (Hrsg.), Legislatures in Comparative Perspective, New York 1973; Gerhard Loewenberg / Samuel C. Patterson, Comparing Legislatures, Boston 1979.

23 Vgl. zum Beispiel Gerald R. Boynton / Gerhard Loewenberg, Der Bundestag im Bewusstsein der Öffentlichkeit 1951-1959, in: PVS, 14. Jg. (1973), H. 1, S. 3 - 25; Dietrich Herzog / Manfred Hirner, Abgeordnete und Bürger. Ergebnisse einer Befragung der Mitglieder des Deutschen Bundestages und der Bevölkerung, Opladen 1990; Werner Patzelt, Abgeordnete und Bürger. Erwartungen und Enttäuschungen, in: Ulrich Sarcinelli (Hrsg.), Öffentlichkeitsarbeit der Parlamente. Politikvermittlung zwischen Public Relations und Parlamentsdidaktik, Baden-Baden 1994, S. 85 - 105; Werner J. Patzelt, a.a.O. (Fn. 20); ders., Warum verachten die Deutschen ihr Parlament und lieben ihr Verfassungsgericht? Ergebnisse einer vergleichenden demoskopischen Studie, in: ZParl, 36. Jg. (2005), H. 3, S. 517 - 538; Suzanne S. Schüttemeyer, Bundestag und Bürger im Spiegel der Demoskopie, Opladen 1986. 
Jahren durchgeführte Umfragen Daten über das Vertrauen der Deutschen zum Deutschen Bundestag und zu Politikern. Sie erlauben es nicht nur, das Niveau und die Entwicklung dieser Einstellungen in West- und Ostdeutschland zu analysieren, sondern ermöglichen es zudem, unsere Befunde aus dem Jahr 2005 in den Kontext der längerfristigen Entwicklung des Vertrauensverhältnisses der Bevölkerung zu Parlament und Politikern einzuordnen.

Die in der Zeit vor der Wiedervereinigung Deutschlands erhobenen Daten über das Vertrauen der Westdeutschen zum Bundestag vermitteln ein völlig anderes Bild, als es für die Vereinigten Staaten beschrieben wurde. Demnach waren die Vertrauensbeziehungen zwischen den Bürgern und dem Parlament intakt (vgl. Abbildung 1). Eine breite Mehrheit vertraute dem Bundestag, und die leichten periodischen Schwankungen folgten dem Wahlzyklus. Schon kurz nach der Wiedervereinigung zeichnete sich jedoch ein Wandel ab. Nach 1990 nahm das Vertrauen ins Parlament in Westdeutschland geradezu dramatisch ab und erreichte im Jahr 1997 den tiefsten Stand, der in Umfragen jemals gemessen wurde. Erstmals seit der Erhebung entsprechender Daten bekundeten mehr Bürger Misstrauen als Vertrauen in das Parlament. Der Regierungswechsel 1998 brachte zwar einen vorübergehenden Wiederanstieg des Vertrauens auf das Niveau der frühen 1990er Jahre, dem seit 2002 aber ein erneuter Einbruch folgte. Allerdings wurden in Westdeutschland zu keinem Zeitpunkt so niedrige Werte gemessen wie in den Vereinigten Staaten. Abgesehen von den Jahren 1997, 2004 und 2006 gab es in den alten Bundesländern stets mehr Vertrauen als Misstrauen.

Für die Bürger in den neuen Bundesländern brachte die Wiedervereinigung die Notwendigkeit mit sich, sich in einem neuen politischen System zurechtzufinden. Auf diese

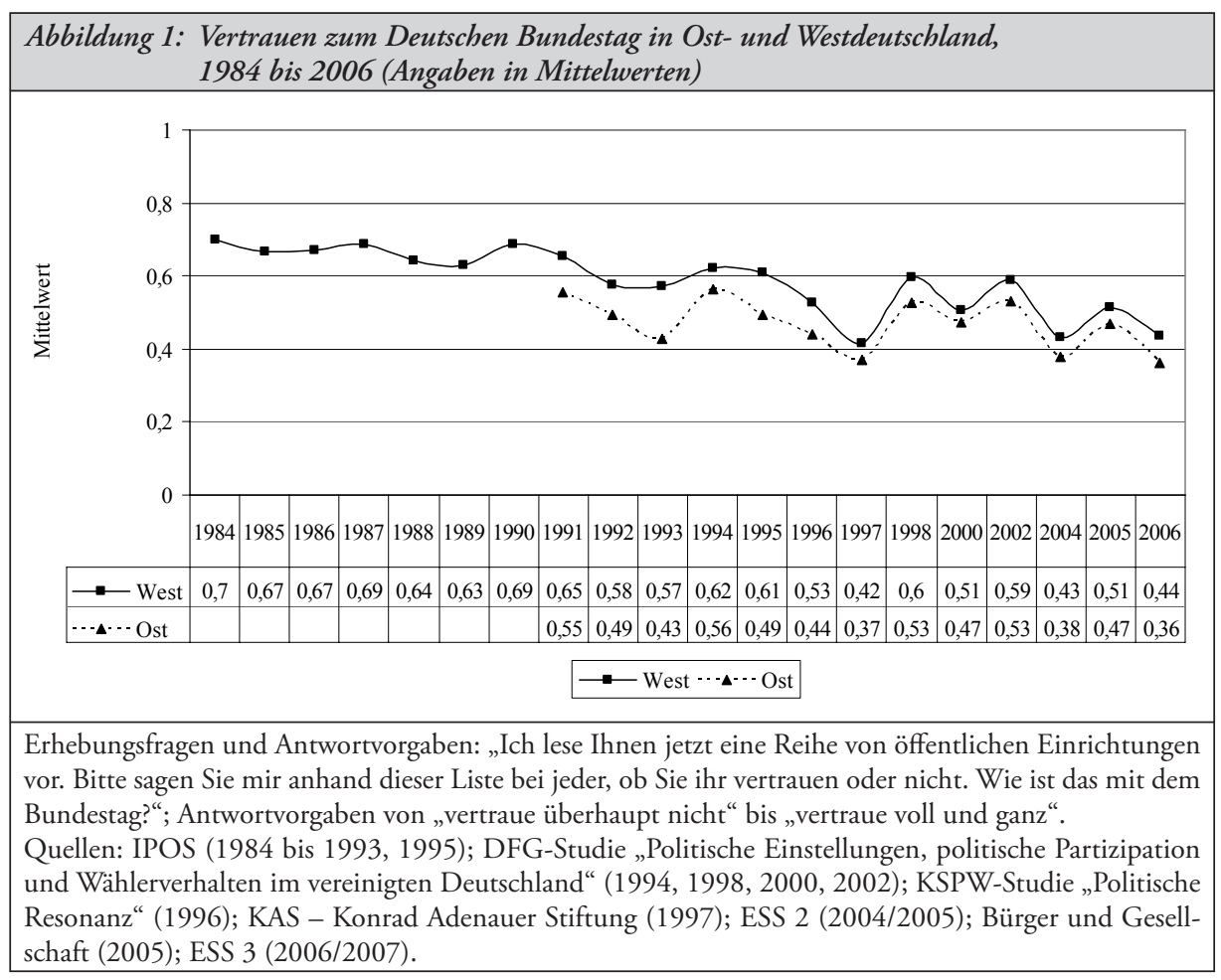


Veränderung reagierten sie zunächst verhalten positiv. In der ersten im Jahr 1991 in Ostdeutschland durchgeführten Umfrage war das Vertrauen zum Bundestag zwar geringer als in den alten Ländern, aber es gab deutlich mehr positive als negative Bewertungen. In den folgenden Jahren entwickelten sich die Einstellungen auf einem etwas niedrigeren Niveau ähnlich wie im westlichen Landesteil. Allerdings besteht ein entscheidender Unterschied im Vertrauen der westdeutschen und der ostdeutschen Bevölkerung zum Bundestag. Während in den alten Ländern fast ausnahmslos mehr positive als negative Einstellungen gemessen wurden, überwog in den neuen Ländern fast immer die Kritik gegenüber der Zustimmung. Nur in den Wahljahren 1994, 1998 und 2002 sowie 1991 war dies nicht der Fall. Unabhängig davon fiel das Vertrauen der ostdeutschen Befragten zum Bundestag fast ausnahmslos höher aus als in den Vereinigten Staaten.

Daten über das Vertrauen der Bürger zu den Abgeordneten wurden in Deutschland nur selten erhoben, jedoch liegen mehrere Umfragen vor, die Informationen über das Vertrauen

\begin{tabular}{|c|c|c|c|c|}
\hline $\begin{array}{l}\text { Abbildung 2: Vertrauen zu Politikern in Ost- und Westdeutschland, } 1994 \text { bis } 2006 \\
\text { (Angaben in Mittelwerten) }\end{array}$ \\
\hline
\end{tabular}


zu Politikern generell enthalten. Wie Abbildung 2 zeigt, ist dies erheblich schwächer ausgeprägt als das Vertrauen zum Bundestag, wobei sich die Einstellungen der west- und ostdeutschen Befragten praktisch nicht voneinander unterscheiden. In den Wahljahren des Zeitraums 1994 bis 2006 war in der Regel ein annäherndes Gleichgewicht zwischen positiven und negativen Einstellungen zu verzeichnen. In den dazwischen liegenden Erhebungen überwog dagegen das Misstrauen.

Ähnlich wie es Hibbing und Theiss-Morse für die USA festgestellt hatten, werden in Deutschland die Abgeordneten nicht grundsätzlich positiver bewertet als das Parlament. Im Vergleich mit den Politikern oder den Abgeordneten als Gruppe findet der Bundestag in der deutschen Öffentlichkeit deutlich mehr Zustimmung.

Die von uns als Datengrundlage der folgenden Analysen benutzte Umfrage verfolgte das Ziel, das Vertrauen zu den Kerninstitutionen des parlamentarischen Regierungssystems der Bundesrepublik differenzierter als üblich zu erheben. Aus diesem Grunde enthielt sie zusätzlich zu den üblichen Fragen nach dem Vertrauen zum Parlament und zur Regierung auch solche nach den Regierungs- und Oppositionsfraktionen, den politischen Parteien, dem Regierungschef und den Wahlkreisabgeordneten. Eine weitere, nicht in der betreffenden Itembatterie enthaltene Einzelfrage bezog sich auf das Vertrauen zu den Abgeordneten als Gruppe. ${ }^{24}$ Bevor wir uns der Beantwortung der Frage zuwenden, ob die Bundesbürger dem Deutschen Bundestag als Institution und den Abgeordneten in unterschiedlichem Maße vertrauen, ist zu klären, ob sie zwischen diesen Objekten überhaupt eine Unterscheidung vornehmen. Aus den bereits erwähnten Gründen ist dies in den europäischen Parteiendemokratien keineswegs selbstverständlich. Eine zur Klärung dieser Frage durchgeführte Faktorenanalyse lieferte keine eindeutige Antwort. Demnach ist im Orientierungssystem der Bundesbürger eine Grunddisposition vorhanden, den Institutionen und Akteuren der parlamentarischen Parteiendemokratie zu vertrauen. Wenn die Bevölkerung überhaupt zwischen verschiedenen Vertrauensobjekten differenziert, dann verläuft die Trennlinie nicht zwischen der Institution und den Abgeordneten, sondern zwischen parteienstaatlichen oder repräsentativen (Oppositionsfraktionen, politische Parteien, Abgeordneten als Gruppe und Wahlkreisabgeordnete) und gouvernementalen Einrichtungen (Bundesregierung, Bundeskanzler und Regierungsfraktionen). Diese Einstellungsstruktur spiegelt die Realität des "Neuen Dualismus" wider. Das Vertrauen zu den Abgeordneten kann also in den Augen der Bundesbürger nicht generell vom Vertrauen zum Deutschen Bundestag getrennt werden. Allerdings weist das Parlamentsvertrauen noch eine zusätzliche Komponente auf, denn es ist auch mit dem Vertrauen zur Regierung, zum Regierungschef und den Regierungsfraktionen verbunden. Die in den amerikanischen Studien unterstellte, aber statistisch nicht überprüfte Unterscheidung zwischen der Bewertung des Kongresses und der Abgeordneten $^{25}$ lässt sich in Deutschland nur bedingt bestätigen. Die deutsche Öffentlichkeit nimmt

24 Daneben kamen zur Bewertung des Parlaments und der Regierung noch weitere Vertrauensitems zum Einsatz, die hier aber im Hinblick auf die Äquivalenz der Messungen nicht ausgewertet werden.

25 Diese Unterscheidung stützt sich bei Glenn R. Parker / Roger H. Davidson, a.a.O. (Fn. 2), John R. Hibbing / Elisabeth Theiss-Morse, a.a.O. (Fn. 3) und mehreren anderen Autoren auf einen Vergleich der Randverteilungen der betreffenden Einstellungen. Kritisch äußert sich zu diesem Vorgehen Richard Born, The Shared Fortunes of Congress and Congressmen: Members May Run from Congress, but they Can't Hide, in: The Journal of Politics, 52. Jg. (1990), H. 4, S. 1223 1241, S. 1224. 
den Bundestag und seine Abgeordneten als Handlungseinheit wahr, sieht aber zugleich einen Zusammenhang zwischen dem Vertrauen zum Bundestag und zur Regierung.

Der nachgewiesene Zusammenhang zwischen dem Vertrauen zum Bundestag und den Abgeordneten schließt keineswegs aus, dass die Bürger diesen Trägern des politischen Lebens unterschiedliches Vertrauen entgegenbringen und dass dies auf unterschiedlichen Erwägungen basiert. Die in Tabelle 1 enthaltenen Daten belegen, dass die meisten Befragten dem Parlament und den Abgeordneten mit einer Mischung aus Vertrauen und Skepsis begegnen. Bei den positiven Bewertungen zeigt sich ein etwas anderes Bild als in den Vereinigten Staaten. Den höchsten Vertrauenskredit räumen die Bürger dem Bundestag ein, gefolgt von den Wahlkreisabgeordneten und den Abgeordneten im Allgemeinen. Dieses Muster zeigt sich in den alten Bundesländern klarer als in den neuen, in denen die Bürger dem Bundestag und den Wahlkreisabgeordneten etwa in gleichem Maße vertrauen und nur die Abgeordneten als Gruppe schlechter bewerten. Bei den negativen Urteilen stimmt die Rangfolge der Objekte in West- und Ostdeutschland überein. Am wenigsten misstrauen die Menschen dem Bundestag als Institution, die zweite Position nehmen die Wahlkreisabgeordneten ein, und das Schlusslicht bilden die Abgeordneten im Allgemeinen. Generell bringen die Ostdeutschen dem Parlament und den Abgeordneten weniger Vertrauen entgegen als die westdeutsche Bevölkerung. Ein weiterer wichtiger Unterschied in der Bewertung des Bundestages und der Wahlkreisabgeordneten betrifft die Sicherheit des Urteils über die Vertrauenswürdigkeit dieser Objekte. Über sein Vertrauen zu den Wahlkreisabgeordneten macht jeder fünfte Befragte keine Angaben, beim Bundestag und den Abgeordneten im Allgemeinen sind es weniger als fünf Prozent (tabellarisch nicht ausgewiesen). Dies bestätigt die Annahme, dass ein großer Teil der Bundesbürger die Wahlkreisabgeordneten nicht kennt beziehungsweise gar keine Vorstellung von ihrer Vertrauenswürdigkeit hat.

\begin{tabular}{|l|c|c|c|c|c|c|c|c|c|}
\hline \multirow{2}{*}{ Tabelle 1: Vertrauen zum Bundestag und zu den Abgeordneten in den alten und neuen } \\
Bundesländern \\
\hline
\end{tabular}

Erhebungsfragen und Antwortvorgaben: Ich nenne Ihnen noch einmal einige Einrichtungen und Personen des politischen Lebens in Deutschland. Bitte sagen Sie mir zu jeder, ob Sie ihr voll und ganz, eher, teils / teils, eher nicht oder überhaupt nicht vertrauen. Wie ist das mit dem Deutschen Bundestag? Und wie ist das mit der Bundesregierung, den Oppositionsfraktionen im Deutschen Bundestag, den Regierungsfraktionen im Deutschen Bundestag, den politischen Parteien, dem Bundeskanzler, den Abgeordneten Ihres Wahlkreises im Deutschen Bundestag?

Vertraue voll und ganz, vertraue eher, teils / teils, vertraue eher nicht, vertraue überhaupt nicht, weiß nicht, keine Antwort.

Und nun zu den Abgeordneten des Deutschen Bundestages. Bitte sagen Sie mir wieder zu jeder dieser Aussagen, ob Sie ihr stark, etwas, teils / teils, eher nicht oder überhaupt nicht zustimmen.

Die Bundestagsabgeordneten sind vertrauenswürdige Menschen.

Stimme voll und ganz zu, stimme eher zu, teils / teils, stimme eher nicht zu, stimme überhaupt nicht zu, weiß nicht, keine Antwort.

Quelle: „Bürger und Gesellschaft“, 2005 (gültige N = 2316, Alte Bundesländer 1851, Neue Bundesländer 465), repräsentativ gewichtet. 
Für Deutschland muss die von Parker und Davidson gestellte Frage, warum die Bürger die Abgeordneten mehr lieben als das Parlament, somit umformuliert werden. Anders als die US-Bürger misstrauen die Deutschen dem Parlament weniger als den Wahlkreisabgeordneten und den Abgeordneten im Allgemeinen. Insbesondere in den alten Bundesländern übertrifft die Wertschätzung der Institution deutlich die der Akteure. Offen bleibt damit jedoch, welche Faktoren hierfür maßgeblich sind und ob das Vertrauen zum Parlament von anderen Faktoren beeinflusst wird als das Vertrauen zu den Abgeordneten.

\subsection{Bestimmungsfaktoren des Vertrauens der Bundesbürger zum Bundestag und zu den Abgeordneten}

Menschen vertrauen ihrer sozialen und politischen Umwelt aus einer Reihe von Gründen. In der Vergangenheit gesammelte positive Erfahrungen begründen die Bereitschaft, einen Vertrauenskredit für die Zukunft zu gewähren. Das Bewusstsein, zu einer sozialen Gruppe zu gehören beziehungsweise bestimmte Überzeugungen zu teilen, ist ebenso eine Quelle des Vertrauens wie die positive Bewertung von Persönlichkeitsmerkmalen eines Interaktionspartners. In der empirischen Forschung erwiesen sich zwei Einstellungen als besonders wichtige Prädiktoren des Vertrauens zu politischen Institutionen und Akteuren: die Bewertung ihrer Leistung und die Überzeugung von ihrer Responsivität. ${ }^{26}$ Die unterschiedliche Leistungsbewertung der Wahlkreisabgeordneten und des US-Kongresses hatte nach Parker und Davidson auch das differierende Vertrauen zu diesen beiden Akteurseinheiten hervorgerufen. Bei ihrem Urteil über den Kongress und die Abgeordneten hatten die Amerikaner jedoch unterschiedliche Arten von Leistungen im Blick. ${ }^{27}$ Hibbing und Theiss-Morse machten für die Kritik am US-Kongress vor allem die unzulängliche Verwirklichung der Prinzipien der Gerechtigkeit (equity) und der Effizienz verantwortlich. ${ }^{28}$ Möglicherweise sind aber die Zuschreibung von Fairness und Vertrauenswürdigkeit zwei Seiten derselben Medaille. Aus den bisher präsentierten Befunden über das Niveau des Vertrauens zum Parlament und zu den Abgeordneten ergibt sich somit die Frage, ob die Bundesbürger dem Bundestag als Institution mehr vertrauen als den Wahlkreisabgeordneten, weil sie ihn für responsiver halten und seine Arbeit besser bewerten oder ob sie beide Objekte nach unterschiedlichen Kriterien bewerten. ${ }^{29}$

26 Vgl. Bas Denters / Oscar Gabriel / Mariano Torcal, Political Confidence, in: Jan W. van Deth / José Ramón Montero / Anders Westholm (Hrsg.), Citizenship and Involvement in European Democracies. A Comparative Analysis, London 2007, S. 66 - 88; Oscar W. Gabriel/ Melanie Walter-Rogg, Social Capital and Political Trust, in: Heiner Meulemann (Hrsg.), Social Capital in Europe. Similarity of Countries and Dissimilarity of People? Multilevel Analyses of the European Social Survey 2002, Leiden / Boston 2008, S. 219 - 250; Oscar W. Gabriel / Sonja Zmerli, Politisches Vertrauen. Deutschland in Europa, in: APuZ, B30 / 31 (2006), S. 8 - 15. Siehe auch den Beitrag von Joachim Behnke in diesem Heft der ZParl zum Einfluss von Werten auf Einstellungen zu politischen Institutionen.

27 Vgl. Glenn R. Parker / Roger H. Davidson, a.a.O. (Fn. 2).

28 Vgl. John R. Hibbing / Elisabeth Theiss-Morse, a.a.O. (Fn. 3).

29 Erhebungsfragen und Indizes: Leistungsbewertung: „Ich würde jetzt gerne noch einmal auf die politischen Einrichtungen zurückkommen, über die wir eben gesprochen haben. Würden Sie sagen, dass diese Einrichtungen ihre Arbeit sehr gut, ziemlich gut, teils gut/teils schlecht, ziemlich schlecht oder sehr schlecht machen? 1. Der Deutsche Bundestag? 2. Die Abgeordneten Ihres 
Nun hängt das Vertrauen zu Parlament und Abgeordneten nicht ausschließlich davon ab, ob die Bevölkerung diese Träger des politischen Lebens für effizient und responsiv hält. In der Forschung über die Vertrauenskrise der politischen Institutionen der repräsentativen Demokratie finden sich etliche Hinweise auf weitere Determinanten des Vertrauens. ${ }^{30}$ Besondere Aufmerksamkeit fand in der öffentlichen Debatte der Beitrag der Medienberichterstattung zum Aufbau und Verfall des politischen Vertrauens. Nach den von Michael Robinson in die empirische Forschung eingebrachten „Videomalaise“- Hypothese untergräbt die negativistische Berichterstattung der Massenmedien, insbesondere des Fernsehens, das Vertrauen der Bürger zur Politik. ${ }^{31}$ Dass der empirische Nachweis eines derartigen Effekts bisher misslang, hat unter anderem mit der unzulänglichen Operationalisierung des Konstrukts „Medieneinfluss“ zu tun. Die meisten empirischen Studien beschränkten sich nämlich darauf, die politische Wirkung der Intensität der Mediennutzung zu untersuchen und lassen die wahrgenommene inhaltliche Tendenz der Berichterstattung ebenso unberücksichtigt wie deren Glaubwürdigkeit. ${ }^{32} \mathrm{Da}$ die Bürger in modernen Gesellschaften die politischen Institutionen und Akteure fast nur noch vermittelt über die Massenmedien wahrnehmen, erscheint der Einfluss der Medienberichterstattung auf das politische Vertrauen hochgradig plausibel. Es ist also anzunehmen, dass eine als negativ wahrgenommene Medienberichterstattung über das Parlament beziehungsweise die Abgeordneten das Vertrauen zu diesen Akteuren entsprechend negativ beeinflusst. ${ }^{33}$ Zusätzlich vermuten wir einen unterschiedlichen Effekt der Fernseh- und der Presseberichterstattung auf das Vertrauen zu den Abgeordneten und zum Bundestag. Die bundesweit ausgerichtete Berichterstattung des Fernsehens dürfte die Einstellungen zum Bundestag stärker beeinflussen als jene zu den Wahlkreisabgeordneten; bei der stärker lokal orientierten Berichterstattung der meisten Zeitungen dürfte es sich umgekehrt verhalten.

Wahlkreises im Deutschen Bundestag?“ Bewertung der Responsivität: Additiver Index zum Bundestag: „Ich lese Ihnen jetzt einige Aussagen über den Bundestag und die Bundesregierung vor. Sagen Sie mir bitte zu jeder, wie sehr Sie ihr zustimmen oder wie sehr Sie diese Aussage ablehnen. 1. Der Deutsche Bundestag orientiert sich bei seinen Entscheidungen nicht am Wohl der gesamten Bevölkerung, sondern an den Interessen einzelner einflussreicher Gruppen. 2. Der Deutsche Bundestag berücksichtigt bei seinen Entscheidungen nicht, was die Bürger denken." Additiver Index zu den Abgeordneten: „Und nun zu den Abgeordneten des Deutschen Bundestages. Bitte sagen Sie mir wieder zu jeder dieser Aussagen, ob Sie ihr stark, etwas, teils/teils, eher nicht oder überhaupt nicht zustimmen. 1. Die Abgeordneten des Deutschen Bundestages bemühen sich nicht besonders darum, einen engen Kontakt zur Bevölkerung zu halten. 2. Die Abgeordneten des Deutschen Bundestages sind nur an Wählerstimmen interessiert, für die Ansichten der Bevölkerung interessieren sie sich nicht."

30 Vgl. die Angaben in Fn. 26.

31 Vgl. Michael Robinson, Public Affairs Television and the Growth of Malaise: The Case of the Selling of the Pentagon, in: American Political Science Review, 70. Jg. (1976), H. 2, S. $409-432$.

32 Vgl. zum Beispiel Seymour Martin Lipset / William Schneider, The Confidence Gap. Business, Labor and Government in the Public Mind, New York / London 1983; Pippa Norris, The Impact of Television on Civic Malaise, in: Susan J. Pharr / Robert D. Putnam (Hrsg.), Disaffected Democracies. What's Troubling the Trilateral Countries, Princeton 2000, S. $231-251$.

33 Erhebungsfragen: „Welchen Eindruck hatten Sie davon, wie in den Zeitungsartikeln [im Fernsehen] über die jeweiligen Einrichtungen oder Personen des politischen Lebens berichtet wurde: War die Berichterstattung sehr positiv, eher positiv, ausgewogen, eher negativ oder sehr negativ? Wie ist es mit ... 1. dem Deutschen Bundestag? 2. den Abgeordneten Ihres Wahlkreises im Deutschen Bundestag?"“ 
Immer wieder wurden auch Spekulationen über die Bedeutung des politischen Wissens für die Einstellungen zur Politik angestellt; allerdings waren die Wirkungsannahmen uneinheitlich. Aus der Perspektive normativer Demokratie- und Sozialisationstheorien lässt sich die Annahme ableiten, politisches Wissen fördere das politische Vertrauen. ${ }^{34}$ Implizit liegt diese Sichtweise auch Werner Patzelts Analyse der Parlamentarismuskritik in der Bundesrepublik zugrunde, die seiner Auffassung nach zu einem erheblichen Teil auf eine Fehlperzeption der Funktionsprinzipien der parlamentarischen Demokratie zurückzuführen ist. ${ }^{35}$ Hingegen findet man mitunter auch die Annahme, politisches Wissen fördere zynische Einstellungen, weil man mit zunehmendem kognitiven Engagement die Schwächen des politischen Betriebes besser durchschaue. ${ }^{36}$ Die kognitive Komponente der Einstellungen zum Bundestag und zu den Wahlkreisabgeordneten wurde durch mehrere Fragen nach dem politischen Wissen und dem Interesse für diese Objekte erhoben. ${ }^{37}$

Auch wenn in der Literatur eine Reihe weiterer Determinanten des politischen Vertrauens zu finden sind, wird hier nur der Einfluss der bisher genannten Größen untersucht. Aufgrund der Datenlage bleiben die Einstellungen zur Gruppe der Abgeordneten unberücksichtigt, denn für diese liegen die benötigten Informationen nicht im gleichen Detaillierungsgrad vor wie für die Wahlkreisabgeordneten und den Bundestag als Institution. Unser Modell zur Schätzung des Vertrauens enthält somit die folgenden Prädiktorgrößen: Die Bewertung der Arbeit des Bundestages beziehungsweise der Wahlkreisabgeordneten, die Einstellung zu deren Responsivität, das Interesse am Bundestag beziehungsweise dem Wahlkreisabgeordneten sowie das Wissen über sie und die Wahrnehmung einer positiven Tendenz der Zeitungs- und TV-Berichterstattung über diese Objekte.

Wie Tabelle 2 zeigt, lässt sich das Vertrauen zum Bundestag und zu den Wahlkreisabgeordneten durch das beschriebene Modell gleich gut schätzen und basiert auf ähnlichen, nur graduell unterschiedlichen Erwägungen. Das Vertrauen zum Bundestag hängt wie das zu den Abgeordneten am stärksten von der Bewertung ihrer Arbeit ab. Bei beiden Objekten handelt es sich um die mit weitem Abstand wichtigste Determinante des politischen Vertrauens, die jedoch die Einstellung zum Bundestag noch etwas stärker beeinflusst als das

34 Vgl. Norman H. Nie / Jane Junn / Kenneth Stehlik-Barry, Education and Democratic Citizenship in America, Chicago / London 1996.

35 Vgl. Werner J. Patzelt, a.a.O. (Fn. 20).

36 Vgl. Giacomo Sani, The Political Culture of Italy. Continuty and Change, in: Gabriel A. Almond I Sidney Verba (Hrsg.), The Civic Culture Revisited. An Analytic Study, Newbury Park 1980, S. $273-324$.

37 Erhebungsfragen und Indizes: Wissen über Bundestag (additiver Index aus der Anzahl der korrekten Antworten): „Können Sie mir bitte sagen, über welche Fragen der Deutsche Bundestag im letzten Jahr Beschlüsse gefasst hat? 1. Abschaffung der Wehrpflicht 2. Einführung einer Praxisgebühr 3. Erhöhung der Mineralölsteuer.“ „Können Sie mir jetzt bitte noch sagen, wer zurzeit Bundestagspräsident(in) ist? Wolfgang Thierse, Ulla Schmidt oder Franz Müntefering?“ „Und wer ist Fraktionsvorsitzender beziehungsweise Fraktionsvorsitzende der CDU/CSU? Friedrich Merz, Wolfgang Bosbach oder Angela Merkel?“ Wissen über Abgeordnete (offene Frage): „Wer hat bei der letzten Bundestagswahl im September 2002 in Ihrem Wahlkreis das Direktmandat gewonnen? Können Sie mir bitte den Namen und die Parteizugehörigkeit dieser Person sagen?" Interesse an den Objekten: „Wie stark interessieren Sie sich persönlich für die Arbeit der folgenden Einrichtungen: sehr stark, ziemlich stark, teils / teils, ziemlich wenig oder sehr wenig? Wie ist es mit ... 1. dem Deutschen Bundestag? 2. den Abgeordneten Ihres Wahlkreises im Deutschen Bundestag?" 
Vertrauen zu den Abgeordneten. Auch in den USA hatte sich eine starke Leistungsabhängigkeit des politischen Vertrauens gezeigt, wobei die für die Bewertung des Kongresses und der Abgeordneten maßgeblichen Leistungskriterien nicht identisch waren. Die in Tabelle 3 berichteten Effekte der Performanzbewertung lassen keine Aussage darüber zu, was die Bürger im Einzelnen meinen, wenn sie mit der Arbeit des Bundestages und der Abgeordneten zufrieden sind. Möglicherweise stellen sie die Qualität der politischen Entscheidungen in den Vordergrund der Bewertung des Bundestages und gewichten für die Bildung ihrer Einstellung zu den Abgeordneten die Wahlkreisarbeit stärker. Definitive Aussagen hierüber lassen sich auf der Basis unserer Daten nicht machen. Neben dem stärkeren Effekt trägt auch die positivere Einschätzung der Arbeit des Bundestages (Bundestag: 21 Prozent, Abgeordnete: 12 Prozent) dazu bei, dass das Parlament als Institution in der deutschen Öffentlichkeit mehr Vertrauen findet als die Wahlkreisabgeordneten.

Im Vergleich mit der Leistungsbewertung spielt die Einstellung zur Responsivität zwar eine untergeordnete Rolle. Sie übt aber gleichwohl einen originären Einfluss auf das Vertrauen zum Parlament und den Abgeordneten aus, der für die Institution doppelt so stark ausfällt wie für die Akteure. Unterschiede im Niveau dieser Einstellung fallen dagegen kaum ins Gewicht. Die Überzeugung, das Parlament verhalte sich responsiv, ist bei den Befragten nur geringfügig weiter verbreitet als die analoge Einstellung zu den Abgeordneten (17 beziehungsweise 13 Prozent). Da das Responsivitätsgefühl das Vertrauen zum Parlament aber ungleich stärker beeinflusst als das zu den Wahlkreisabgeordneten, trägt auch dies dazu bei, das höhere Niveau des Vertrauens zum Bundestag als Institution zu erklären.

\begin{tabular}{|c|c|c|c|c|}
\hline \multicolumn{5}{|c|}{ 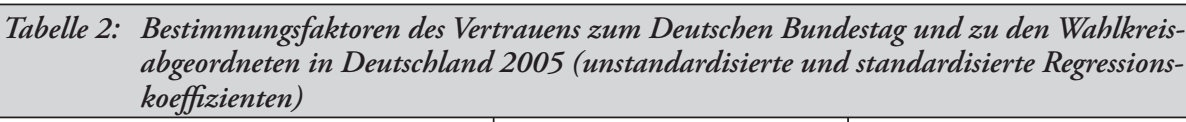 } \\
\hline & \multicolumn{2}{|c|}{ Bundestag } & \multicolumn{2}{|c|}{ Wahlkreisabgeordnete } \\
\hline & $\mathrm{B}$ & Beta & $\mathrm{B}$ & Beta \\
\hline Leistungsbewertung &, $51^{\mathrm{a}}$ & 43 & $40^{\mathrm{a}}$ & 36 \\
\hline Bewertung der Responsivität &, $16^{\mathrm{a}}$ &, 17 &, $07^{\mathrm{b}}$ & ,08 \\
\hline Interesse am Objekt &, $03^{\text {n.s. }}$ &, 03 &, $10^{\mathrm{a}}$ & , 11 \\
\hline Wissen über das Objekt &, $06^{\mathrm{b}}$ &, 05 &, $01^{\text {n.s. }}$ & ,02 \\
\hline $\begin{array}{l}\text { Wahrgenommene Tendenz der Zeitungs- } \\
\text { berichterstattung }\end{array}$ &, $07^{\mathrm{b}}$ & ,06 & $25^{\mathrm{a}}$ & ,22 \\
\hline $\begin{array}{l}\text { Wahrgenommene Tendenz der Fernseh- } \\
\text { berichterstattung }\end{array}$ &, $22^{\mathrm{a}}$ &, 18 &, $17^{\mathrm{a}}$ &, 14 \\
\hline Konstante &, $00^{\text {n.s. }}$ & &, $03^{\text {n.s. }}$ & \\
\hline $\mathrm{R}^{2}$ &, $41^{\mathrm{a}}$ & &, $42^{\mathrm{a}}$ & \\
\hline $\mathrm{N}$ & 1826 & & 836 & \\
\hline \multicolumn{5}{|c|}{$\begin{array}{l}\text { Hinweise: }{ }^{\text {a }} \text { t- bzw. F-Wert auf dem } 99,9 \% \text {-Niveau statistisch signifikant; }{ }^{\mathrm{b}} \text { t- bzw. F-Wert auf dem } 99,0 \\
\% \text {-Niveau statistisch signifikant; }{ }^{\mathrm{c}} \mathrm{t} \text { - bzw. F-Wert auf dem 95,0 \%-Niveau statistisch signifikant; }{ }^{\text {n.s. }} \text { t- } \\
\text { bzw. F-Wert auf dem 95,0 \%-Niveau statistisch insignifikant. } \\
\text { Quelle: „Bürger und Gesellschaft“, 2005, nach Bevölkerungsanteil der alten und neuen Bundesländer } \\
\text { gewichtet. }\end{array}$} \\
\hline
\end{tabular}

Anders als in den Vereinigten Staaten generieren die Einstellungen zur Arbeit des Parlaments und der Abgeordneten sowie zu ihrer Responsivität in Deutschland einen Vertrau- 
ensvorsprung des Bundestages vor den Abgeordneten. Die Bürger bewerten den Bundestag als Institution auf beiden Dimensionen nicht nur positiver als die Wahlkreisabgeordneten, sondern sie setzen diese Einstellungen auch stärker in politisches Vertrauen um.

Neben den theoretisch gut begründeten und mehrfach geprüften Annahmen über die Zusammenhänge zwischen Vertrauen, Leistungsbewertung und Responsivität enthält unser Schätzmodell weitere Erklärungsfaktoren, über deren Bedeutung für das Entstehen und die Aufrechterhaltung des politischen Vertrauens nur wenige Erkenntnisse vorliegen. Es handelt sich hierbei um Indikatoren der kognitiven Anteilnahme der Bürger an der Parlamentsarbeit und der von ihnen perzipierten Tendenz der Medienberichterstattung über diese. Nach den in Tabelle 2 enthaltenen Daten spielen das Wissen über die parlamentarische Arbeit beziehungsweise die politische Konstellation im Wahlkreis sowie das Interesse an diesen Sachverhalten keine maßgebliche Rolle dafür, ob die Bürger dem Bundestag oder den Abgeordneten vertrauen. Wenn man nur die direkten und unabhängigen Effekte betrachtet, tragen diese Faktoren wenig dazu bei, das unterschiedliche Niveau des Vertrauens zum Bundestag und zu den Wahlkreisabgeordneten zu erklären. Auch die Tatsache, dass das Interesse gering und das Wissen der Bevölkerung mäßig ist, begrenzt die Relevanz kognitiver Faktoren für das Vertrauen zum Parlament und seinen Mitgliedern. Ein großes oder sehr großes Interesse am Bundestag bekunden lediglich 18 Prozent der Befragten. Bei den Wahlkreisabgeordneten wird mit acht Prozent nicht einmal ein halb so großer Wert erreicht. Kein oder geringes Wissen über die Arbeit des Bundestages liegt bei circa 30 Prozent der Befragten vor. 42 Prozent können weder den Namen ihres Wahlkreisabgeordneten noch die Parteizugehörigkeit des im Wahlkreis erfolgreichen Kandidaten nennen beziehungsweise machen auf den ersten Blick falsche Angaben hierzu. Da sowohl die Effektstärke als auch das Niveau der Prädiktoren das Niveau des Vertrauens beeinflussen, leisten beide Parameter einen Beitrag dazu, dass kognitive Orientierungen weitgehend irrelevant für die Bildung und Aufrechterhaltung des politischen Vertrauens sind.

Im Gegensatz zu den bisherigen Forschungsergebnissen belegen die in Tabelle 2 enthaltenen Daten einen substanziell bedeutenden Einfluss der perzipierten Medienberichterstattung. Diese erweist sich nach der Bewertung der Arbeit des Parlaments beziehungsweise des Abgeordneten als wichtigster Bestimmungsfaktor des politischen Vertrauens. Ihr Einfluss auf das Vertrauen zum Parlament fällt etwa gleich stark aus wie der Effekt der Responsivitätsbewertung, während das Vertrauen zu den Wahlkreisabgeordneten sogar noch stärker durch Medieneffekte geprägt ist als durch die Überzeugung, die Abgeordneten verhielten sich responsiv. Wie erwartet, variieren die Effekte der Zeitungs- und der Fernsehberichterstattung mit den Adressaten des Vertrauens. Eine positiv wahrgenommene Fernsehberichterstattung stärkt das Vertrauen zum Bundestag, die Printmedien spielen für die Einstellung zum Parlament als Institution eine vernachlässigbare Rolle. Ganz anders stellt sich die Sachlage für das Vertrauen zu den Wahlkreisabgeordneten dar. Es hängt stärker von der Tendenz der Berichterstattung der Zeitungen als von den durch das Fernsehen verbreiteten Botschaften ab. Diese sind nicht unerheblich, aber nicht so wichtig wie die Wahrnehmung der Inhalte der Printmedien. ${ }^{38}$

38 Die Berücksichtigung möglicher Wechselwirkungen zwischen der Häufigkeit der Mediennutzung oder der den Medien zugewiesenen Glaubwürdigkeit verbessert die Erklärungsleistung des Modells nicht (tabellarisch nicht ausgewiesen). 
Die große Bedeutung der perzipierten Medienberichterstattung für die Bildung und Aufrechterhaltung politischen Vertrauens wird erst in ihrem vollen Umfange deutlich, wenn man berücksichtigt, dass im Schätzmodell mit der Bewertung der Leistungen der Abgeordneten und des Parlaments und der Einschätzung der Responsivität dieser Akteure zwei außerordentlich wichtige Bestimmungsfaktoren des politischen Vertrauens konstant gehalten wurden. Eine positiv wahrgenommene Fernsehberichterstattung über den Bundestag fördert somit unabhängig vom eigenen Urteil der Bürger über dessen Arbeit und Responsivität das Vertrauen zum Parlament. Auf das Vertrauen zu den Wahlkreisabgeordneten wirkt sich eine als positiv wahrgenommene Berichterstattung der Zeitungen sogar noch etwas stärker aus. Umgekehrt gilt: Wenn sich erst einmal die Vorstellung herausgebildet hat, dass der Bundestag gute Arbeit leistet und bei seinen Entscheidungen die Interessen der Bürger berücksichtigt, fördern diese Einstellungen selbst gegen eine negative Medienberichterstattung das Vertrauen zum Parlament. Für das Vertrauen zu den Wahlkreisabgeordneten gelten dieselben Überlegungen. Einschränkend ist allerdings festzuhalten, dass diese Medieneffekte nur bei einem Teil der Befragten wirksam wurden, weil circa 15 Prozent die Medien nicht nutzten oder nicht dazu in der Lage waren, die Tendenz ihrer Berichterstattung zu beurteilen. Bei der Bildung von Vertrauen zu den Abgeordneten schlägt dieser Sachverhalt noch viel stärker zu Buche. Nur zehn Prozent der Befragten gaben an, aus der Zeitung oder dem Fernsehen häufig oder sehr häufig Informationen über die Wahlkreisabgeordneten erhalten zu haben; nur jeder zweite konnte Angaben über die Tendenz der Medienberichterstattung über die Wahlkreisabgeordneten machen. Aus diesen Befunden kann man die Folgerung ableiten, dass der Bundestag zumindest einer relativ starken Minderheit der Bundesbürger kognitiv ausgesprochen präsent ist, während nur eine sehr kleine Minderheit der Befragten dazu in der Lage ist, ihr Urteil über die Wahlkreisabgeordneten kognitiv abzusichern.

\section{Zusammenfassung und Folgerungen: Warum die Deutschen ihrem Abgeordneten nicht mehr vertrauen als dem Bundestag}

Viele, aber keineswegs alle US-Bürger vertrauten in den 1970er und 1980er Jahren den Wahlkreisabgeordneten mehr als dem Kongress. In Deutschland war das Vertrauen in den Bundestag im Jahr 2005 nicht besonders groß, aber das in die Wahlkreisabgeordneten war noch geringer. Warum aber vertrauen die Bundesbürger ihren Wahlkreisabgeordneten noch weniger als ihrem Parlament?

Es ist plausibel, die im Vergleich mit der Institution Kongress breitere Vertrauensbasis der US-amerikanischen Abgeordneten auf institutionelle und kulturelle Besonderheiten der Vereinigten Staaten zurückzuführen. Die institutionelle Ausgestaltung des Mandats schafft für die Rolleninhaber dort starke Anreize, der Wahlkreisarbeit in ihrem Tätigkeitsprofil hohe Priorität einzuräumen. Die dadurch bedingte Basisnähe der Volksvertreter und die vermeintliche persönliche Vertrautheit mit ihnen veranlassen viele amerikanische Wähler, den Abgeordneten stärker zu vertrauen als der Legislative. Dieser Vertrauensvorsprung ist allerdings an die Bedingung geknüpft, dass die Bürger die Repräsentanten und Senatoren als Vertreter ihres Wahlkreises wahrnehmen. Sobald sie diese dem „Washingtoner System“ zurechnen, schwindet die Zustimmung und dem Kongress als Institution wird eine größere Wertschätzung zuteil. Diese Bedingungen sind in Deutschland und in anderen europäi- 
schen Parteiendemokratien nicht gegeben. Die Frage, ob die institutionellen Arrangements tatsächlich dafür ausschlaggebend sind, dass die Amerikaner andere Vertrauensbeziehungen zu den Abgeordneten und zum Parlament entwickeln als die Deutschen, lässt sich auf der Basis der vorliegenden Untersuchung nicht entscheiden. Die Annahme ist plausibel, empirisch aber nicht zu belegen. Auch über die möglichen Effekte der unterschiedlichen kulturellen Traditionen beider Länder, insbesondere des amerikanischen Anti-Institutionalismus und der deutschen Staatsgläubigkeit, kann man nur spekulieren.

Relativ eindeutige Aussagen sind dagegen über die individuellen Bestimmungsfaktoren des Vertrauens der Deutschen zum Bundestag und zu den Abgeordneten möglich. Wie Parker und Davidson festgestellt hatten, bewerten die US-Bürger die Abgeordneten nach anderen Kriterien als den Kongress. Da diese Kriterien die Abgeordneten in einem guten und den Kongress in einem schlechten Licht erscheinen ließen, fiel die Einschätzung der parlamentarischen Akteure positiver aus als die der Institution. Hier konnte nicht geprüft werden, ob die Deutschen den Bundestag auf der Basis anderer Standards bewerten als die Wahlkreisabgeordneten. Wir konnten aber zeigen, dass das in den USA für die Bewertung beider Objekte maßgebliche Kriterium der Leistungsfähigkeit auch in Deutschland das Vertrauen zum Parlament und zu den Abgeordneten stark beeinflusst. Es ist für das Vertrauen zur Institution wichtiger als für das Vertrauen zu den Akteuren, zudem bewerten die Bundesbürger die Arbeit des Bundestages weniger negativ als die der Abgeordneten. Ähnliches gilt für die Einschätzung der Bürgernähe des Parlaments und seiner Mitglieder.

Mit anderen Worten: Die Deutschen vertrauen den Wahlkreisabgeordneten noch weniger als der Institution Deutscher Bundestag, weil sie deren Arbeit kritischer bewerten, weil sie diese für weniger bürgernah halten und weil ihnen die Presse ein negativeres Bild von der Arbeit der Abgeordneten vermittelt als von jener des Bundestages. Um allerdings eindeutige Aussagen darüber zu machen, ob diese Unterschiede zu den Charakteristika der Politischen Kultur beider Länder gehören oder ob sie eine Momentaufnahme des Verhältnisses der Bürger zur Politik vermitteln, bedürfte es einer breiteren Datenbasis. 